\title{
Eupatilin rescues ciliary transition zone defects to ameliorate ciliopathy-related phenotypes
}

\author{
Yong Joon Kim, ${ }^{1}$ Sungsoo Kim, ${ }^{2}$ Yooju Jung, ${ }^{2}$ Eunji Jung, ${ }^{1}$ Ho Jeong Kwon, ${ }^{2}$ and Joon Kim ${ }^{1}$ \\ 'Craduate School of Medical Science and Engineering, Korea Advanced Institute of Science and Technology (KAIST), Daejeon, South Korea. ${ }^{2}$ Chemical Genomics Clobal Research Laboratory, Department of \\ Biotechnology, Yonsei University, Seoul, South Korea.
}

\begin{abstract}
Ciliopathies are clinically overlapping genetic disorders involving structural and functional abnormalities of cilia. Currently, there are no small-molecule drugs available to treat ciliary defects in ciliopathies. Our phenotype-based screen identified the flavonoid eupatilin and its analogs as lead compounds for developing ciliopathy medication. CEP290, a gene mutated in several ciliopathies, encodes a protein that forms a complex with NPHP5 to support the function of the ciliary transition zone. Eupatilin relieved ciliogenesis and ciliary receptor delivery defects resulting from deletion of CEP290. In rd16 mice harboring a blinding Cep290 in-frame deletion, eupatilin treatment improved both opsin transport to the photoreceptor outer segment and electrophysiological responses of the retina to light stimulation. The rescue effect was due to eupatilinmediated inhibition of calmodulin binding to NPHP5, which promoted NPHP5 recruitment to the ciliary base. Our results suggest that deficiency of a ciliopathy protein could be mitigated by small-molecule compounds that target other ciliary components that interact with the ciliopathy protein.
\end{abstract}

\section{Introduction}

Mutations in a number of genes required for the formation or function of primary cilia have been identified in ciliopathies, such as Joubert syndrome, Meckel-Gruber syndrome, and nephronophthisis (1). In addition, ciliopathy mutations interfering with opsin trafficking through the connecting cilium of photoreceptors account for a significant proportion of hereditary retinal dystrophies, including Leber congenital amaurosis (LCA) $(2,3)$. Protein products of genes mutated in these ciliopathies commonly act as a component or an interactor of transition zone complexes that form a barrier structure at the ciliary base to control transit of molecules into and out of the cilium (4).

Although individual ciliopathies are rare genetic disorders, collectively, the prevalence of ciliopathies may reach 1 in 1,000 births (5). Currently, there are no approved medications available for the treatment of most ciliopathies. CEP29O is a gene frequently mutated in several ciliopathies and LCA (6). CEP290 gene augmentation therapy using viral vectors has been shown to ameliorate LCA-related phenotypes in human cell and mouse models $(7,8)$. Oligonucleotide-mediated splicing correction has also been shown to rescue LCA phenotypes in the induced pluripotent stem cell-derived optic cups of patients harboring a CEP290 intronic mutation (9). In this study, we aimed to identify lead compounds for the treatment of CEP290-related ciliopathies through cellbased compound library screening.

Conflict of interest: YK, SK, YJ, HK, and JK have filed a patent application in South Korea for ciliogenesis-promoting low-molecular-weight compounds and a therapeutic agent for ciliopathy (application number: 10-2017-0171656)

Submitted: December 13, 2017; Accepted: May 31, 2018.

Reference information: / Clin Invest. 2018;128(8):3642-3648.

https://doi.org/10.1172/JCI99232

\section{Results and Discussion}

Human RPE1 cells stably expressing EGFP-tagged Smoothened (RPE1-Smo-EGFP) were used for the screen to simplify the visualization of primary cilia and ciliary membrane protein transport. First, we generated a RPE1-Smo-EGFP cell line harboring frameshift mutations in exon 2 of CEP29O (Supplemental Figure 1A; supplemental material available online with this article; https://doi.org/10.1172/ JCI99232DS1), using the CRISPR/Cas9 system (10). CEP290 depletion was confirmed by immunoblotting and immunofluorescence staining (Supplemental Figure 1, B and C). CEP290 ${ }^{\text {null }}$ cells showed defects in ciliogenesis induced by serum starvation (Figure $1 \mathrm{~A}$ and Supplemental Figure 1, D and E). Transient expression of mCherrytagged CEP290 restored cilium assembly in CEP290 ${ }^{\text {null }}$ cells, indicating that the ciliogenesis defect was caused by CEP290 loss (Supplemental Figure 1F).

We screened a library of 2,789 synthetic and natural compounds and identified 22 compounds that increased the number of ciliated cells in the CEP290 ${ }^{\text {null }}$ context (Figure 1B, Supplemental Table 1, and Supplemental Figure 2). The compounds showing the strongest rescue effect were 4 structural analogs of plant flavonoids: eupatilin, jaceosidin, lysionotin, and quercetin. They share the core molecular structure of a 15-carbon skeleton, which consists of 2 phenyl rings and a heterocyclic ring (Figure 1C). The rescue effect of the flavonoids was compared by a dose-response analysis, and eupatilin was selected for further analyses (Figure 1D). To determine whether eupatilin could exert a general rescue effect on the loss of ciliopathy proteins, we treated CEP290wT RPE1 cells with eupatilin after siRNA-mediated knock down of several ciliopathy genes $(4,11,12)$. Serum starvation-induced ciliogenesis was not rescued by eupatilin in cells depleted of the selected ciliopathy genes, whereas eupatilin facilitated ciliogenesis in cells transfected with negative control siRNA (Figure 1E). 


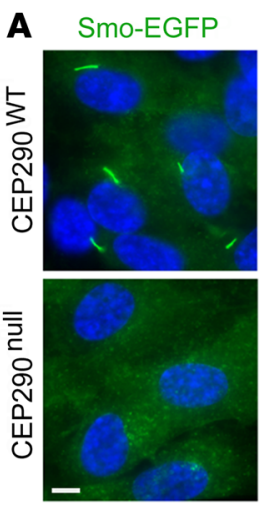

D

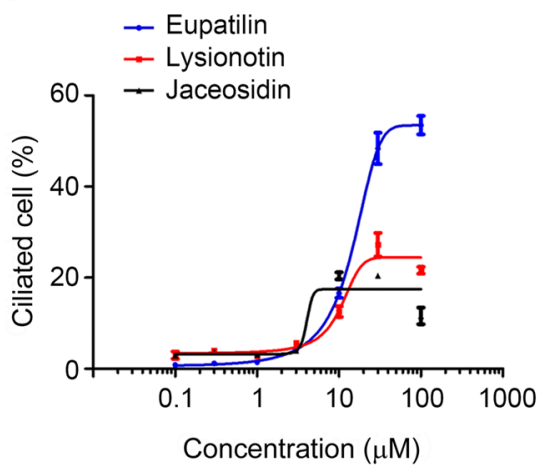

B

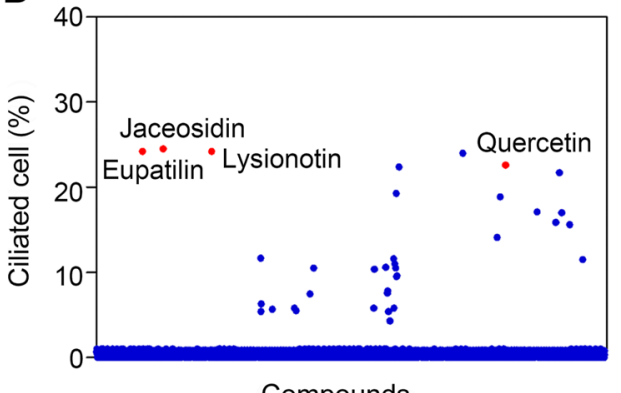

Compounds
C<smiles>COc1ccc(-c2cc(=O)c3c(O)c(OC)c(OC)cc3o2)cc1OC</smiles>

Eupatilin<smiles>O=c1c(O)c(-c2ccc(O)c(O)c2)oc2cc(O)cc(O)c12</smiles>

Lysionotin<smiles></smiles>

Jaceosidin<smiles>COc1cc(-c2cc(=O)c3c(O)c(OC)cc(O)c3o2)ccc1O</smiles>

Quercetin

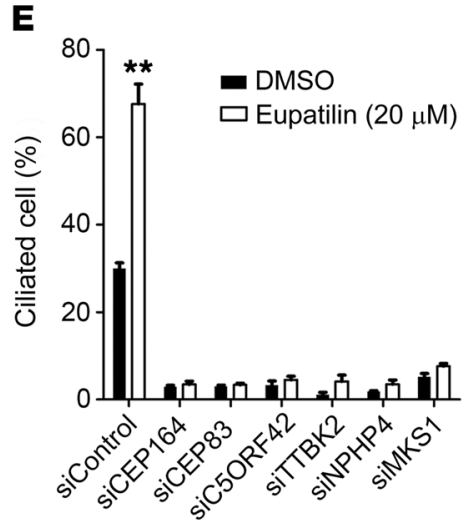

Figure 1. Identification of compounds that rescue ciliogenesis defects caused by CEP290 knockout. (A) Defects in serum starvation-induced ciliogenesis in Smo-EGFP+ CEP290 ${ }^{\text {null }}$ RPE1 cells. Scale bar indicates $5 \mu \mathrm{m}$. Nuclei were marked with DAPI (blue). (B) Screening of chemical compounds that promote cilium assembly in Smo-EGFP+ ${ }^{2}$ EP290 ${ }^{\text {null }}$ RPE1 cells. (C) Structures of plant flavonoids identified as screen hits. (D) Flavonoid dose-response curves of Smo-EGFP+ CEP290 ${ }^{\text {null }}$ RPE1 cells. (E) Effect of eupatilin on serum starvation-induced ciliogenesis in CEP290wT RPE1 cells transfected with the indicated siRNAs. (F) Effect of cytoD and eupatilin on ciliogenesis in CEP290WT RPE1 cells cultured in the presence of $10 \%$ serum. Error bars represent SEM ( $n=3$ independent experiments; ${ }^{* *} P<0.01, t$ test).

This suggests that although eupatilin can promote ciliogenesis in a CEP290 ${ }^{\text {WT }}$ context, the rescue effect of eupatilin is specific for CEP290 loss.

Actin filament destabilizer cytochalasin D (cytoD) has been shown to alleviate ciliogenesis defects in Ift88 mutant embryonic fibroblasts and induce ciliogenesis in RPE1 cells even without serum starvation (13). Unlike cytoD, eupatilin treatment did not induce ciliogenesis in the presence of serum (Figure $1 \mathrm{~F}$ ) and did not alter the structure of the actin cytoskeleton (Supplemental Figure 3A). Moreover, Ki67 expression and YAP nuclear enrichment, which reflect cell proliferation and cilium disassembly gene expression (14), respectively, were only affected by cytoD treatment (Supplemental Figure 3, B-D). Cell proliferation assay validated that eupatilin exerts a lower inhibitory effect on cell proliferation when compared with cytoD (Supplemental Figure 3E). These results suggest that eupatilin and cytoD use different mechanisms to influence the assembly of primary cilia.

Next, we investigated whether known properties of eupatilin were involved in its rescue effect. Eupatilin has been shown to inhibit the PI3K/AKT signaling pathway and activate peroxisome proliferator-activated receptor alpha $(\operatorname{PPAR}-\alpha)(15,16)$. We treated CEP290 ${ }^{\text {null }}$ cells with the PI3K inhibitor BYL-719, the AKT inhibitor MK-2206, and the PPAR- $\alpha$ agonist WY-14643, either alone or together with eupatilin. These compounds did not promote ciliogenesis and the rescue effect of eupatilin was not changed (Supplemental Figure 4). Therefore, eupatilin does not appear to act through PI3K/AKT and PPAR- $\alpha$ to rescue ciliogenesis defects.

To better understand eupatilin-mediated ciliogenesis rescue, we analyzed steps of ciliogenesis in CEP290 ${ }^{\text {null }}$ cells. TTBK2 recruitment to the centrosomal area and CP110 cap removal from the mother centriole are known to occur at the beginning of ciliogenesis $(11,17)$. Both TTBK2 recruitment and CP110 removal appeared normal in CEP290 ${ }^{\text {null }}$ cells (Supplemental Figure 5). Attachment of ciliary vesicles to the distal appendages of the nascent basal body precedes axoneme assembly in RPE1 cells $(12,18)$. We observed an increase in the number of cells exhibiting either a Smo-EGFP ${ }^{+}$ciliary vesicle attached to the basal body or pericentriolar Smo-EGFP ${ }^{+}$vesicles in a CEP290 ${ }^{\text {null }}$ background (Supplemental Figure 6, A and B). These results suggest that the failure of ciliogenesis in CEP290 ${ }^{\text {null }}$ cells occurs after the cap removal and the ciliary vesicle docking. Axoneme assembly requires intraflagellar transport (IFT), a bidirectional motility along axonemal microtubules (19). Basal body recruitment of IFT88, a key component of the retrograde IFT complex, did not noticeably change in CEP290 ${ }^{\text {null }}$ cells (Supplemental Figure 6C). Therefore, the rescue effect of eupatilin may not occur at these early steps of ciliogenesis. 
A

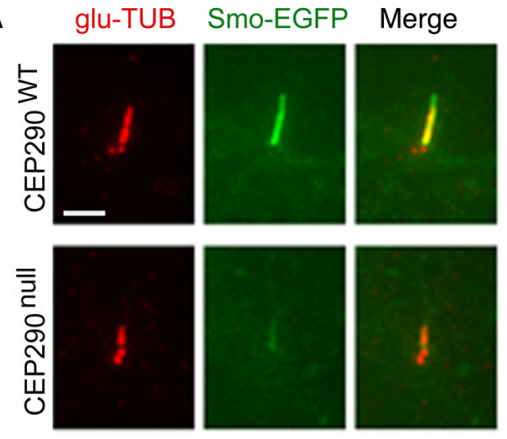

B

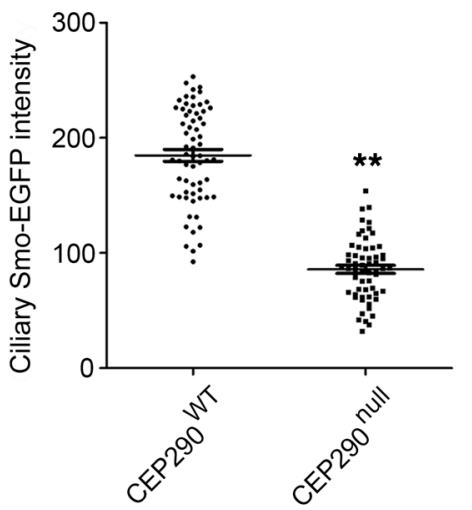

C
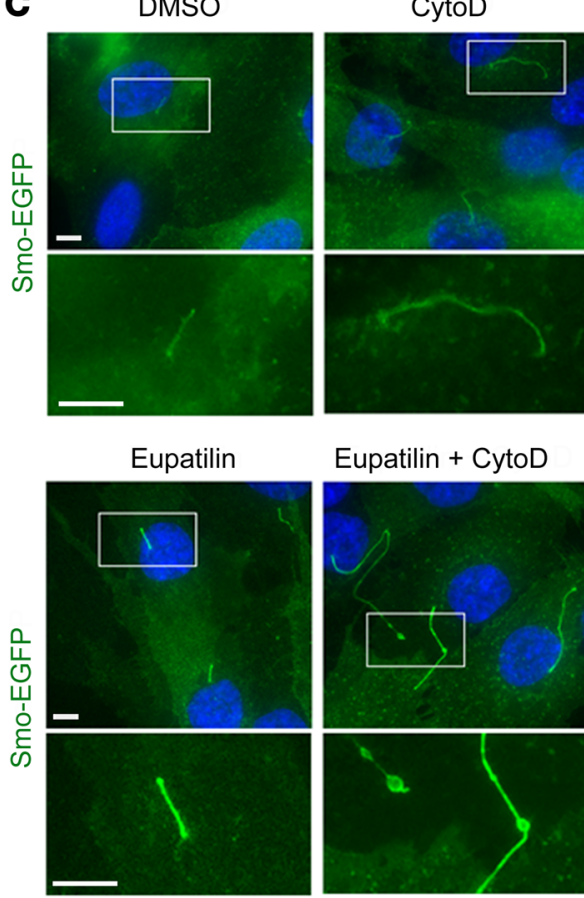
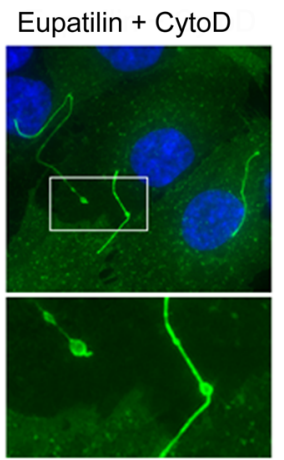

D

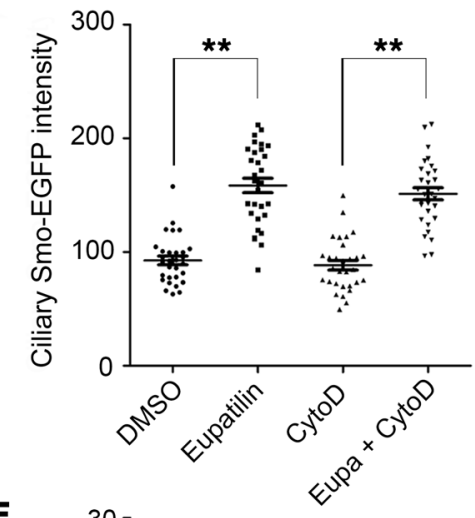

$\mathbf{E}$

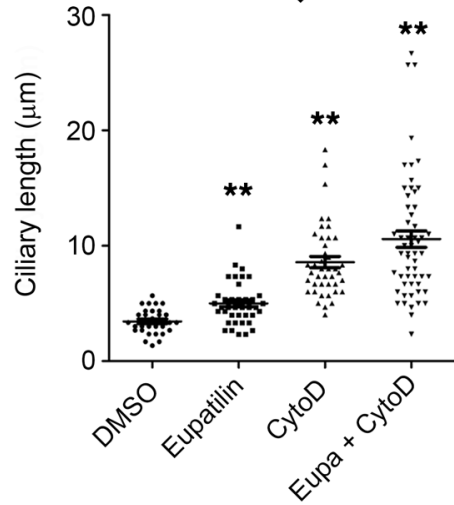

Figure 2. Eupatilin promotes Smo-EGFP transport to the ciliary membrane. (A) Fluorescence micrographs visualizing ciliary Smo-EGFP intensity in CEP290'T and CEP290 ${ }^{\text {null }}$ RPE1 cells. (B) Quantification (arbitrary unit) of the experiment presented in A. (C) Effect of cytoD and eupatilin on ciliary SmoEGFP intensity and cilium elongation in CEP290 null RPE1 cells. Cells were treated with the indicated chemicals for 48 hours in the absence of serum. (D and E) Quantification (arbitrary unit) of the experiment presented in C. Scale bars indicate $5 \mu \mathrm{m}$. Error bars represent SEM (30-60 cells of each condition from 2 independent experiments were analyzed; ${ }^{* *} P<0.01, t$ test).

A small number of CEP290 ${ }^{\text {null }}$ cells formed primary cilia positive for the ciliary markers poly-glutamylated tubulin and ARL13B (Supplemental Figure 1E). However, cilia in the mutant cells exhibited a significant decrease in Smo-EGFP fluorescence intensity when compared with cilia from CEP290 ${ }^{\mathrm{WT}}$ cells (Figure 2, A and B). Notably, eupatilin treatment significantly enhanced the intensity of ciliary Smo-EGFP fluorescence and slightly increased ciliary length in CEP290 ${ }^{\text {null }}$ cells (Figure 2, C-E). By contrast, cytoD did not affect ciliary Smo-EGFP intensity and showed a stronger cilium elongation effect. Combined treatment with eupatilin and cytoD exerted a synergistic effect on cilium elongation, and a subset of elongated cilia exhibited abnormal bulges (Figure 2D). CEP290 has been reported to play a role in the establishment of the ciliary transition zone and the delivery of membranous cargos across the transition zone $(20,21)$. Thus, our results suggest that eupatilin improves the function of the transition zone damaged by CEP290 loss.

We tested whether eupatilin can rescue CEP290-related ciliopathy phenotypes in a mouse model. Homozygous $r d 16$ mice harboring a Cep290 in-frame deletion have been shown to exhibit blindness due to complete degeneration of photoreceptors (22). We first confirmed that daily subcutaneous injection of eupatilin $(0-40 \mathrm{mg} / \mathrm{kg})$ did not interfere with body growth or the structure and electrophysiological function of the retina in CD1 mouse neonates (Supplemental Figure 7 and 8). Next, we injected eupatilin $(40 \mathrm{mg} / \mathrm{kg})$ daily into $r d 16$ neonates for 3 weeks from 3 days after birth. As expected, electroretinograms (ERGs) under dark-adapted conditions (scotopic response) and light-adapted conditions (photopic response) indicated a severe deterioration of rod and cone function in vehicle-injected $r d 16$ homozygotes at postnatal day 23. Remarkably, ERGs of eupatilin-injected $r d 16$ homozygotes showed partial but significant recoveries under light-adapted conditions (Figure 3, A and B). ERGs under dark-adapted conditions were not changed by eupatilin (Supplemental Figure 9A). Consistent with the ERG findings, immunofluorescence analyses revealed that $\mathrm{M}$-opsin trafficking to the outer segment of cone photoreceptors was significantly recovered, whereas rhodopsin trafficking was not rescued in eupatilin-treated $r d 16$ mice (Figure 3, C and D; and Supplemental Figure 9B). These results demonstrate that eupatilin treatment improves the function of cone photoreceptors in Cep290-mutant retinas.

CEP290 forms a complex with the ciliopathy protein NPHP5 and also interacts with other transition zone components including the Meckel syndrome (MKS) protein complex $(20,23)$. TCTN1 and TMEM216 of the MKS complex mediate the link between the transition zone and the ciliary membrane $(4,24)$. Although CEP290 is known to be essential for the assembly of the MKS complex at the transition zone in Caenorhabditis elegans (23), the transition zone localization of TCTN1 and TMEM216 was apparently not disrupted in CEP290 $0^{\text {null }}$ cells (Supplemental Figure 10, A and B). By contrast, as previously reported $(25,26)$, much of the 
A

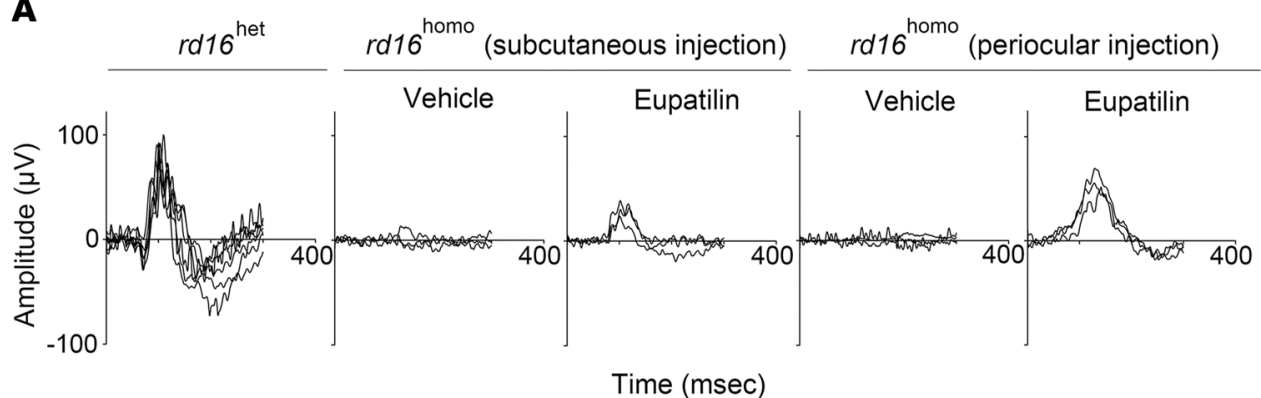

Time (msec)
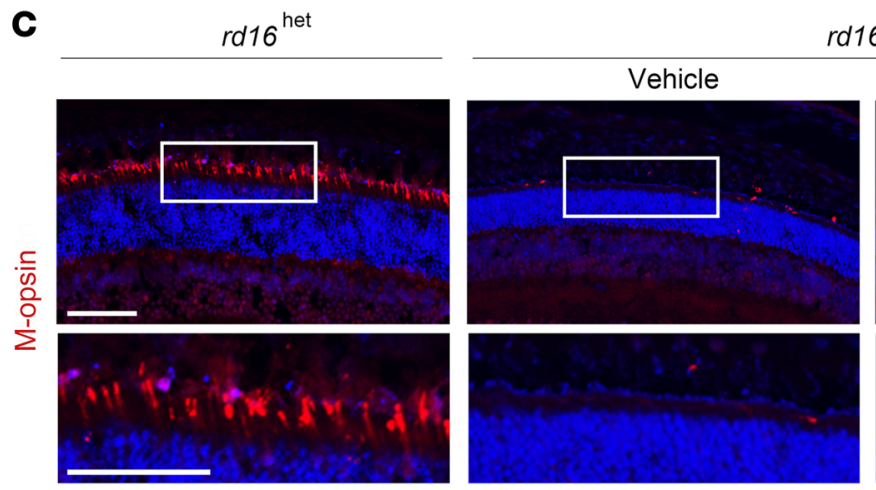

$r d 16^{\text {homo }}$

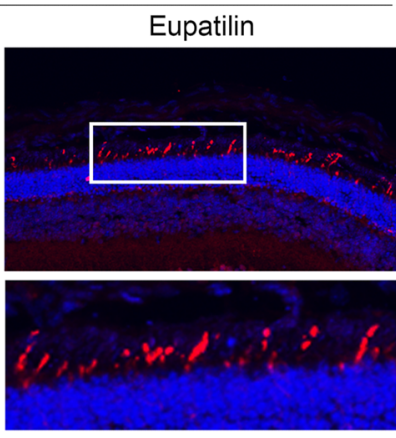

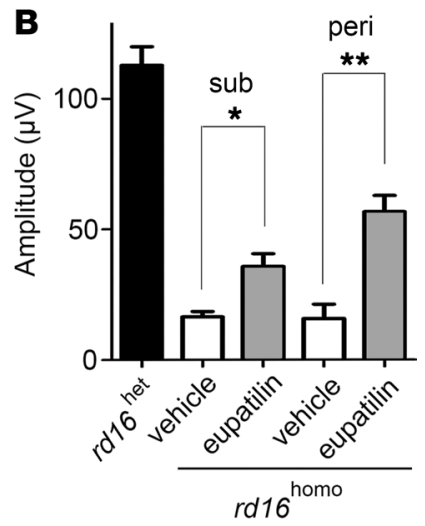

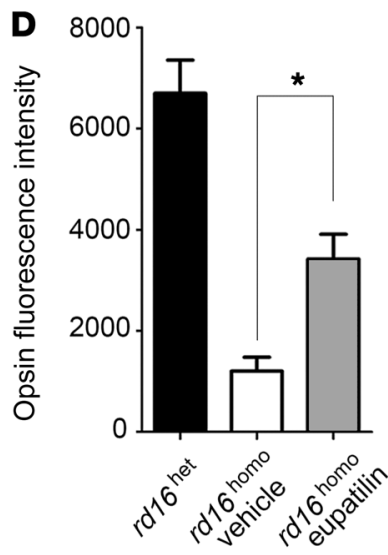

Figure 3. Eupatilin injection ameliorates M-opsin trafficking and electrophysiological response of cone photoreceptors in rd16 mice. (A) Electroretinogram of $r d 16^{\text {het }}$ and $r d 16^{\text {homo }}$ mice at postnatal day 23 under light-adapted conditions after subcutaneous or periocular injection of vehicle or eupatilin (40 $\mathrm{mg} / \mathrm{kg}$ ) for 3 weeks. (B) Amplitude of B-wave in photopic responses. Sub, subcutaneous injection; peri, periocular injection. (C) Fluorescence micrographs visualizing M-opsin in the retina of $r d 16^{\text {het }}$ and $r d 16^{\text {homo }}$ mice injected with vehicle or eupatilin $(40 \mathrm{mg} / \mathrm{kg})$ for 3 weeks. Nuclei were marked with DAPI (blue) (D) Quantification (arbitrary unit) of the experiment presented in C. Scale bars indicate $50 \mu \mathrm{m}$. Error bars represent SEM $\left(n=3\right.$ mice, each group; ${ }^{*} P<0.05$ and ${ }^{* *} P<0.01, t$ test)

transition zone pool of NPHP5 disappeared in CEP290 ${ }^{\text {null }}$ cells (Figure 4, A and B). CEP290 and NPHP5 regulate the integrity of the BBSome, a protein complex involved in ciliary cargo trafficking, and damaged BBSome integrity in CEP290-depleted cells can be rescued by full-length NPHP5 overexpression (20, 21). Importantly, eupatilin treatment restored NPHP5 levels in the transition zone area in CEP290 ${ }^{\text {null }}$ cells (Figure 4, A and B). This suggests that cells treated with eupatilin bypass the requirement for CEP290 in the localization of NPHP5 and facilitated NPHP5 recruitment compensates for the loss of CEP290. Consistent with this idea, the rescue effect of eupatilin in CEP290 ${ }^{\text {null }}$ cells almost disappeared after NPHP5 depletion (Supplemental Figure 11).

NPHP5 possesses distinct CEP290- and calmodulin-binding domains, and the calmodulin-binding domain is dispensable for the localization and function of NPHP5 (25). Previously, eupatilin analog quercetin was shown to interact with and antagonize the $\mathrm{Ca}^{2+}$-calmodulin complex (27). These findings prompted us to test whether eupatilin restored NPHP5 localization in a calmodulin-dependent manner. To test whether eupatilin interacts with calmodulin, we performed a drug affinity responsive target stability (DARTS) experiment $(28,29)$. Eupatilin treatment conferred protease resistance to calmodulin, indicating that there is a physical interaction between eupatilin and calmodulin (Figure
4C). In silico molecular docking analysis also suggested that eupatilin directly interacts with calmodulin (Supplemental Figure 12). Next, we examined whether eupatilin affects the binding affinity between NPHP5 and calmodulin. We performed a coimmunoprecipitation analysis of endogenous NPHP5. Notably, the presence of eupatilin decreased the amounts of calmodulin coprecipitated with NPHP5, whereas coprecipitation of IFT88 was not inhibited (Figure 4D). This shows that eupatilin inhibits the binding between NPHP5 and calmodulin. In addition, we observed an increase in the total level of NPHP5 in response to eupatilin treatment (Figure 4E).

We speculated that calmodulin inhibits the accumulation of NPHP5 at the transition zone in the absence of CEP290. To test this, we performed siRNA-mediated knock down of calmodulin genes. Simultaneous depletion of calmodulin 1, 2, and 3 improved ciliogenesis in CEP290 ${ }^{\text {null }}$ cells (Figure 4F). Moreover, calmodulin knockdown promoted the recruitment of NPHP5 to the transition zone (Figure 4G). The combination of eupatilin treatment and calmodulin knockdown additively increased ciliated cells and cilia length (Figure 4, H-J). Therefore, we conclude that eupatilin facilitates NPHP5 centrosomal localization in a CEP290 $0^{\text {null }}$ context by inhibiting the interaction between calmodulin and NPHP5 (Supplemental Figure 13). Augmented NPHP5 levels may amelio- 


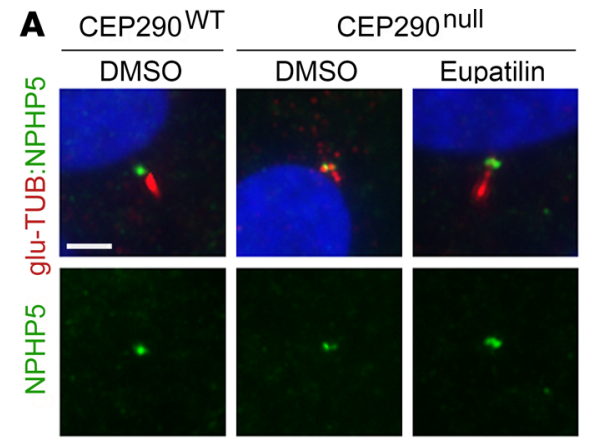

D

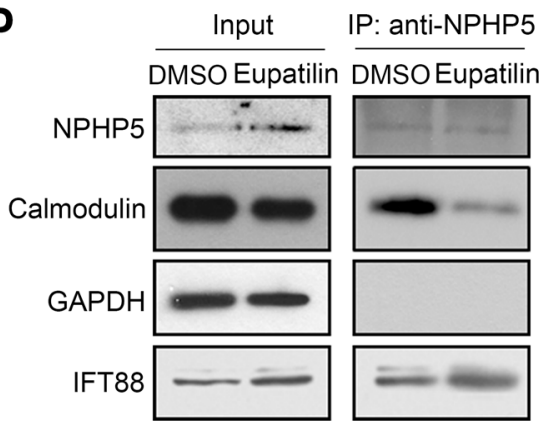

B

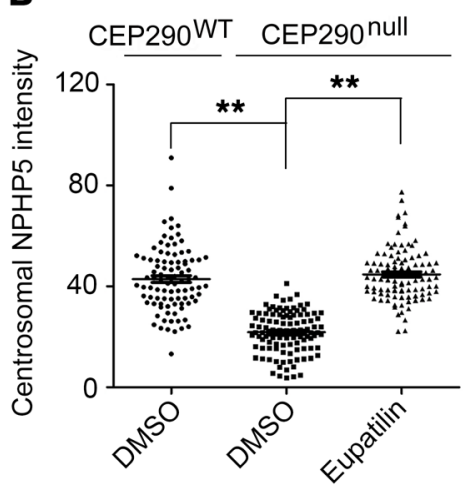

E

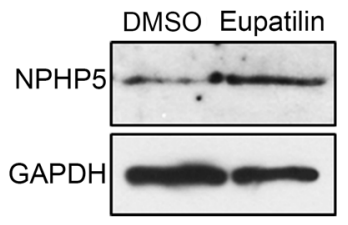

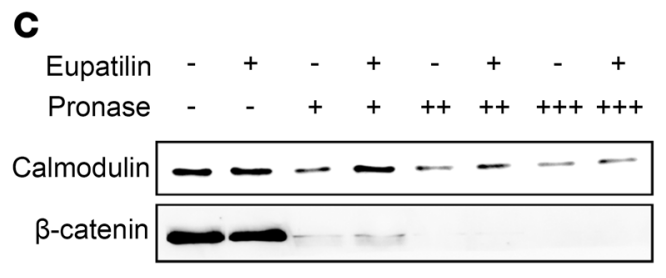

C
G

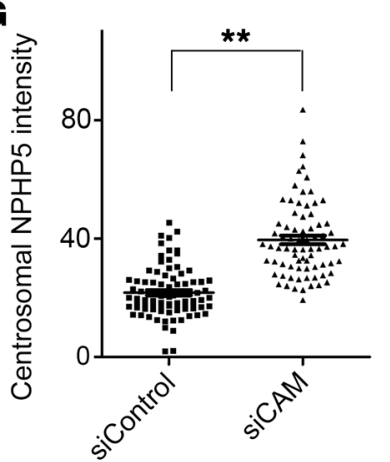

H

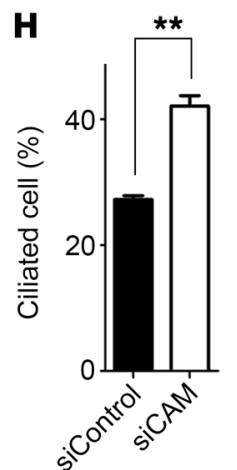

I

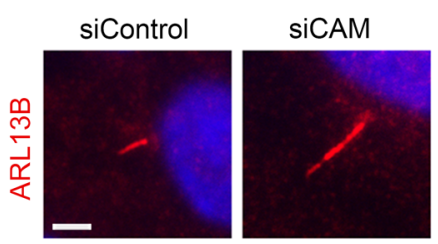

$\mathbf{F}$

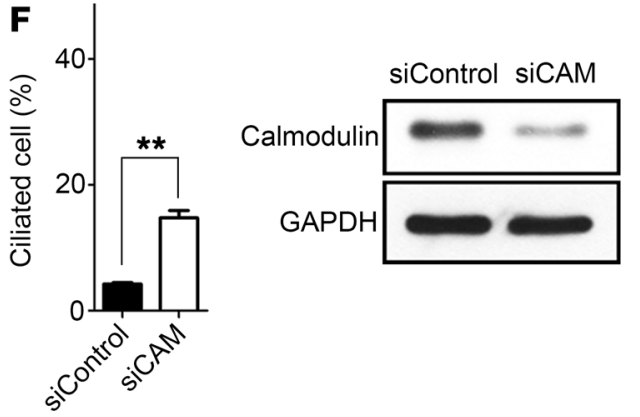

Figure 4. Eupatilin restores centrosomal NPHP5 levels in CEP290null RPE1 cells by inhibiting NPHP5-calmodulin interaction. (A) Fluorescence micrographs visualizing centrosomal localization of NPHP5 in CEP290'T and CEP290 ${ }^{\text {null }}$ RPE1 cells treated with DMSO or eupatilin (20 $\mu$ M) for 24 hours in the absence of serum. (B) Quantification (arbitrary unit) of the experiment presented in A. (C) DARTS-Western blot analysis that shows eupatilin-mediated protection of calmodulin (CAM) against pronase (62.5 ng [+], $250 \mathrm{ng}[++]$, or $1 \mu \mathrm{g}[+++]$ per $100 \mu \mathrm{g}$ total cell protein). (D) Immunoprecipitation of NPHP5 after 48 hours DMSO or eupatilin treatment and coprecipitated calmodulin detected by Western blotting. (E) Immunoblot analysis of NPHP5 after 48 hours DMSO or eupatilin treatment. (F and $\mathbf{G})$ Effect of simultaneous knock down of 3 calmodulin genes on ciliogenesis and centrosomal NPHP5 recruitment in CEP290 null RPE1 cells. Reduction of total calmodulin levels was confirmed by Western blotting. (H-J) Effect of simultaneous knock down of 3 calmodulin genes on ciliogenesis and cilium elongation in CEP290null RPE1 cells treated with $20 \mu \mathrm{M}$ eupatilin. Scale bars indicate $5 \mu \mathrm{m}$. Error bars represent SEM (B, G, J: >50 cells of each condition from 3 independent experiments were analyzed; F, H: $n=3$ independent experiments; ${ }^{* *} P<0.01, t$ test).

rate the transport of membrane proteins through the transition zone. Phenothiazine derivatives and cyclosporin A can function as a calmodulin inhibitor $(30,31)$, and our screen hits include cyclosporin $\mathrm{A}$ and the 2 phenothiazine derivatives acepromazine and methdilazine (Supplemental Table 1 and Supplemental Figure 14). These compounds may also compensate for the loss of CEP290 in a calmodulin-dependent manner.

Previous studies have reported early-onset rapid degeneration of rod photoreceptors in human ciliopathies caused by CEP290 or NPHP5 mutations $(32,33)$. In contrast to early rod losses, the fovea in these patients retained cone nuclei, although cone pho- toreceptors exhibited abnormal morphology of the inner and outer segments (32). Our finding that only cone photoreceptors responded to eupatilin treatment is consistent with the fact that cone photoreceptors are more slowly degenerated than rod photoreceptors $(32,34)$. Significant cone ERG responses were not detected in eupatilin-injected $r d 16$ mice at P30 (not shown). Oxidative damage has been suggested as a major contributing factor to cone cell death following the death of rod cells (35). Thus, it is likely that the diminished effect of eupatilin at P30 was due to the secondary death of cone cells. Despite this limitation, considering that the fovea of the human retina primarily consists of cone pho- 
toreceptors, eupatilin may be used to preserve the central vision of CEP290-associated retinopathy patients.

In conclusion, we identify eupatilin as a lead compound for developing medication for ciliopathies involving retinal degeneration. Eupatilin has been used in clinic as a drug for gastritis and peptic ulcer (36). Therefore, we expect that eupatilin could be repositioned for treating ciliopathy patients carrying CEP290 mutations. Collectively, our study provides evidence that deficiency of a disease protein in recessive genetic disorders can be mitigated by chemical compounds that target other proteins that interact with the disease protein.

\section{Methods}

Quantification and statistical analysis. The percentage of ciliated cells was determined by inspecting at least 200 cells stained with antiARL13B and antiglutamylated tubulin antibodies. Ciliary Smo-EGFP intensity, ciliary length, subcellular YAP localization, and centrosomal NPHP5 levels were measured using the DeltaVision Spectris Imaging System and Image J software (NIH). Data analysis was performed using GraphPad Prism version 6 (GraphPad Software). The 2-tailed Student's $t$ test was used to determine $P$ values throughout the study, and $P<0.05$ was considered statistically significant.

Study approval. Animal experiments were conducted in an animal facility at KAIST. The KAIST IACUC approved the animal care and experimental procedures used in this study (KA2017-20).

For additional information, see Supplemental Methods.

\section{Author contributions}

$\mathrm{JK}$ and HJK conceived and directed the project. YJK and JK designed the screening strategy. YJK and SK performed the screen, and YJK analyzed the screen data. YJK designed and performed the follow-up experiments. YJ and EJ contributed to the experiments. YJK and JK interpreted the results of the follow-up experiments. YJK, HJK, and JK wrote the paper.

\section{Acknowledgments}

We thank Gou Young Koh for providing ERG equipment and Jaeryung Kim for technical support for ERG measurements. This study was supported by Korean Health Industry Development Institute grants (HI12C0014 and HI18C0013) funded by the Korean Ministry of Health and Welfare, Korean National Research Foundation grants (2015M3A9B6027820, 2015K1A1A2028365, 2015M3A9C4076321, and 2015M3A9B6027818) funded by the Korean Ministry of Science and ICT, and the Brain Korea 21Plus Project.

Address correspondence to: Joon Kim, Graduate School of Medical Science and Engineering, KAIST, 291 Daehak-ro, Daejeon 34141, South Korea. Phone: 82.42.350.4242; Email: joonkim@ kaist.ac.kr. Or to: Ho Jeong Kwon, Chemical Genomics Global Research Laboratory, Department of Biotechnology, Yonsei University, 50 Yonsei-ro, Seodaemun-gu, Seoul 120749, South Korea. Phone: 82.2.2123.5883; Email: kwonhj@yonsei.ac.kr.
1. Hildebrandt F, Benzing T, Katsanis N. Ciliopathies. N Engl J Med. 2011;364(16):1533-1543.

2. Wright AF, Chakarova CF, Abd El-Aziz MM, Bhattacharya SS. Photoreceptor degeneration: genetic and mechanistic dissection of a complex trait. Nat Rev Genet. 2010;11(4):273-284.

3. Rachel RA, Li T, Swaroop A. Photoreceptor sensory cilia and ciliopathies: focus on CEP290, RPGR and their interacting proteins. Cilia. 2012;1(1):22.

4. Czarnecki PG, Shah JV. The ciliary transition zone: from morphology and molecules to medicine. Trends Cell Biol. 2012;22(4):201-210.

5. Zaghloul NA, Katsanis N. Mechanistic insights into Bardet-Biedl syndrome, a model ciliopathy. $J$ Clin Invest. 2009;119(3):428-437.

6. den Hollander AI, et al. Mutations in the CEP290 (NPHP6) gene are a frequent cause of Leber congenital amaurosis. Am J Hum Genet. 2006;79(3):556-561.

7. Burnight ER, et al. CEP290 gene transfer rescues Leber congenital amaurosis cellular phenotype. Gene Ther. 2014;21(7):662-672.

8. Zhang W, Li L, Su Q, Gao G, Khanna H. Gene therapy using a miniCEP290 fragment delays photoreceptor degeneration in a mouse model of leber congenital amaurosis. Hum Gene Ther. 2018;29(1):42-50.

9. Parfitt DA, et al. Identification and correction of mechanisms underlying inherited blindness in human iPSC-derived optic cups. Cell Stem Cell. 2016;18(6):769-781.

10. Ran FA, Hsu PD, Wright J, Agarwala V, Scott DA, Zhang F. Genome engineering using the CRISPRCas9 system. Nat Protoc. 2013;8(11):2281-2308.

11. Čajánek L, Nigg EA. Cep164 triggers cilio- genesis by recruiting Tau tubulin kinase 2 to the mother centriole. Proc Natl Acad Sci USA 2014;111(28):E2841-E2850.

12. Joo $\mathrm{K}$, et al. CCDC41 is required for ciliary vesicle docking to the mother centriole. Proc Natl Acad Sci USA. 2013;110(15):5987-5992.

13. Kim J, et al. Functional genomic screen for modulators of ciliogenesis and cilium length. Nature. 2010;464(7291):1048-1051.

14. Kim J, et al. Actin remodelling factors control ciliogenesis by regulating YAP/TAZ activity and vesicle trafficking. Nat Commun. 2015;6:6781.

15. Zhong WF, Wang XH, Pan B, Li F, Kuang L, Su ZX. Eupatilin induces human renal cancer cell apoptosis via ROS-mediated MAPK and PI3K/AKT signaling pathways. Oncol Lett. 2016;12(4):2894-2899.

16. Jung $Y$, et al. Eupatilin with PPAR $\alpha$ agonistic effects inhibits TNF $\alpha$-induced MMP signaling in HaCaT cells. Biochem Biophys Res Commun. 2017;493(1):220-226.

17. Goetz SC, Liem KF, Anderson KV. The spinocerebellar ataxia-associated gene Tau tubulin kinase 2 controls the initiation of ciliogenesis. Cell. 2012;151(4):847-858.

18. Molla-Herman A, et al. The ciliary pocket: an endocytic membrane domain at the base of primary and motile cilia. JCell Sci. 2010;123(Pt 10):1785-1795.

19. Rosenbaum JL, Witman GB. Intraflagellar transport. Nat Rev Mol Cell Biol. 2002;3(11):813-825.

20. Barbelanne M, Hossain D, Chan DP, Peränen J, Tsang WY. Nephrocystin proteins NPHP5 and Cep290 regulate BBSome integrity, ciliary trafficking and cargo delivery. Hum Mol Genet. 2015;24(8):2185-2200.

21. Shimada $H$, et al. In vitro modeling using ciliop- athy-patient-derived cells reveals distinct cilia dysfunctions caused by CEP290 mutations. Cell Rep. 2017;20(2):384-396.

22. Chang $\mathrm{B}$, et al. In-frame deletion in a novel centrosomal/ciliary protein CEP290/NPHP6 perturbs its interaction with RPGR and results in early-onset retinal degeneration in the rd16 mouse. Hum Mol Genet. 2006;15(11):1847-1857.

23. Li C, et al. MKS5 and CEP290 dependent assembly pathway of the ciliary transition zone. PLOS Biol. 2016;14(3):e1002416.

24. Lee JH, et al. Evolutionarily assembled cisregulatory module at a human ciliopathy locus. Science. 2012;335(6071):966-969.

25. Barbelanne M, Song J, Ahmadzai M, Tsang WY. Pathogenic NPHP5 mutations impair protein interaction with Cep290, a prerequisite for ciliogenesis. Hum Mol Genet. 2013;22(12):2482-2494.

26. Sang L, et al. Mapping the NPHP-JBTS-MKS protein network reveals ciliopathy disease genes and pathways. Cell. 2011;145(4):513-528.

27. Nishino $H$, Naitoh $E$, Iwashima $A$, Umezawa K. Quercetin interacts with calmodulin, a calcium regulatory protein. Experientia. 1984;40(2):184-185.

28. Pai MY, et al. Drug affinity responsive target stability (DARTS) for small-molecule target identification. Methods Mol Biol. 2015;1263:287-298.

29. Chang J, Kim Y, Kwon HJ. Advances in identification and validation of protein targets of natural products without chemical modification. Nat Prod Rep. 2016;33(5):719-730.

30. Weiss B, Prozialeck W, Cimino M, Barnette MS, Wallace TL. Pharmacological regulation of calmodulin. Ann N Y Acad Sci. 1980;356:319-345.

31. Colombani PM, Robb A, Hess AD. Cyclosporin A 
binding to calmodulin: a possible site of action on T lymphocytes. Science. 1985;228(4697):337-339.

32. Cideciyan AV, et al. Cone photoreceptors are the main targets for gene therapy of NPHP5 (IQCB1) or NPHP6 (CEP290) blindness: generation of an all-cone Nphp6 hypomorph mouse that mimics the human retinal ciliopathy. Hum Mol Genet. 2011;20(7):1411-1423.
33. Ronquillo CC, Hanke-Gogokhia C, Revelo MP, Frederick JM, Jiang L, Baehr W. Ciliopathyassociated IQCB1/NPHP5 protein is required for mouse photoreceptor outer segment formation. FASEB J. 2016;30(10):3400-3412.

34. Farjo R, et al. Retention of function without normal disc morphogenesis occurs in cone but not rod photoreceptors. JCell Biol. 2006;173(1):59-68.
35. Usui S, et al. NADPH oxidase plays a central role in cone cell death in retinitis pigmentosa. JNeurochem. 2009;110(3):1028-1037.

36. Seol SY, Kim MH, Ryu JS, Choi MG, Shin DW, Ahn BO. DA-9601 for erosive gastritis: results of a double-blind placebo-controlled phase III clinical trial. World J Gastroenterol. 2004;10(16):2379-2382. 